\title{
Active Suspension Control of Full-car Systems without Function Approximation
}

\author{
Jing Na, Member, IEEE, Yingbo Huang*, Member IEEE, Qianqian Pei, Xing Wu, Guanbin Gao and Guang Li, \\ Member, IEEE
}

\begin{abstract}
This paper proposes a new control approach for full-car active suspension systems with unknown nonlinearities. The main advantage of this approach is that the uncertainties and nonlinearities in the system can be handled without using any function approximator (e.g. neural networks (NNs), fuzzy logic systems (FLSs)), and the associated online adaptation. Hence, the heavy computational cost and sluggish learning phase to achieve convergence can be remedied. To maintain the transient and steady-state suspension responses, a coordinate suspension error transformation with prescribed performance functions (PPF) is adopted. Then an approximation-free control (AFC) is developed to achieve stabilization of the transformed system so as to retain predefined suspension response. Extreme Value Theorem is used together with Lyapunov theorem to prove the stability and convergence of the closed-loop control system. To validate the proposed method and show its practical applicability, a dynamic simulator is built by using a commercial vehicle software, Carsim, where an E-SUV type vehicle is configured to describe realistic vehicle dynamics. Simulation results reveal that the proposed control can achieve better suspension performance and require less model information compared with some existing approaches.

Index Terms-Active suspension control, Full-car system, Prescribed performance control, Nonlinear dynamics.
\end{abstract}

\section{INTRODUCTION}

$\mathrm{S}$ USPENSION systems have always played an essential role in the automotive products due to its ability to guarantee the passengers' ride comfort and driving safety (i.e. road holding and suspension stroke limitation) [1,2]. According to different mechanical configures, vehicle suspension systems can be classified into three types: passive suspension, semi-active suspension [3-5] and active suspension [6, 7]. Although passive suspension has been widely used in commercial vehicles due to its low cost and simple structure, its ability to absorb excitations induced by the road conditions is restricted by the fixed spring and damper dynamics. Hence, recent studies have been made toward the developments of semi-active suspension systems [3-5] and active suspension systems [6, 7], which are able to achieve considerably improved suspension performance over passive suspensions [8]. Specifically, active suspension system uses extra actuators together with passive suspension elements

This work was supported by the National Natural Science Foundation of China under Grants 61573174, and a joint PhD Scholarship between Chinese Scholarship Council (CSC) and Queen Mary University of London under Grant 201708530252. (Corresponding author: Yingbo Huang).

Jing Na, Yingbo Huang, Xing Wu and Guanbin Gao are with the Faculty of Mechanical and Electrical Engineering, Kunming University of Science and technology, Kunming, 650500, China. (e-mail: najing25@163.com; Yingbo_Huang@126.com; xingwu@aliyun.com; gbgao@163.com).

Qianqian Pei is with the Faculty of Mechanical and Electrical Engineering, Yunnan Land And Resources Vocational College, Kunming, 652501, China. (e-mail: 18487142740@163.com)

Guang $\mathrm{Li}$ is with the School of Engineering and Materials Science, Queen Mary University of London E1 4NS, UK (e-mail: g.li@qmul.ac.uk). (e.g. springs and dampers) and thus can effectively dismiss forces from the road excitation [9]. However, active suspension has not been widely used in the commercial products yet because of its high energy demand and increased cost. In this respect, the development of advanced control strategies for active suspension systems has been recognized as one of the most promising pathways to achieve cost-reduction and safety enhancements, and thus has attracted significant attentions in both academics and engineering fields [9-13].

In the active suspension system designs, several performance requirements should be considered. Apart from eliminating the uninterrupted disturbance from the road roughness, the ride comfort, road holding and suspension deflection limitation should be considered. To manage the potential contradictions between these requirements, some advanced control methods have been tailored for active suspension applications, e.g. multi-objective control[14], adaptive control[15], backstepping control [16] and preview control [17]. In the aforementioned studies, a critical assumption is that all dynamics of the studied suspension systems should be known accurately and even should be linear. This assumption may not be true in the realistic vehicle suspension systems (e.g. the springs, dampers and actuators used for suspension have nonlinear behaviors), which makes these model based approaches less effective.

To accommodate unknown nonlinearities in the control systems, function approximators, such as neural networks (NNs) and fuzzy logic systems (FLSs) [7, 13, 18-21], have been incorporated into adaptive control designs, where the unknown weights of NNs or FLSs can be online updated via adaptive laws designed to minimize the control errors [22-24]. This methodology allows proving the closed-loop stability by means of the Lyapunov theorem. Although it is mathematically elegant, it is found that practical implementation of function approximation based adaptive control schemes is still not fully mature for commercial products due to their complex structure and demanding computational cost. Specifically, it is still a nontrivial task to tune the parameters used in these adaptive control methods. Moreover, the online learning of the function approximators needs fairly long time to achieve convergence, and the potentially sluggish convergence rate and/or large overshoot during the transient stage may create risks for operation safety of adaptive control systems [25].

It is also noted that quantitatively study transient response of nonlinear control systems is generally difficult. Recently, Bechlioulis and Rovithaks introduced a new control framework $[26,27]$ to guarantee both the transient and steady-state control convergence responses within a predefined boundary. The key idea is to incorporate a prescribed performance function (PPF) and the associate error transformation into the control designs. 
This idea has been subsequently tailored for other control system designs [28-30]. Nevertheless, in these PPF based control designs, function approximators are still needed to accommodate the unknown system dynamics. To address this issue, an approximation-free control (AFC) [31] was further introduced to address tracking control of nonlinear systems. In this method, NNs and FLSs are not required, while both the transient and steady-state control performances are guaranteed. Hence, this technique provides a potentially new constructive methodology for nonlinear control system designs, which is also useful for active suspensions [21].

On the other hand, it is also found from active suspension control literatures that most of existing results focus on quartercar or half-car dynamics only, while only few results have been reported for full-car dynamics $[10,32]$. In fact, the full-car suspension system has multi-inputs-multi-outputs and certain couplings between the vertical, roll and pitch motions, which makes the corresponding control design difficult. Hence, the aim of this paper is to introduce a new control design for full-car active suspension systems with unknown dynamics. The main merit is to further tailor the concept of AFC $[9,31]$ and extend this idea to full-car suspension applications, where the vertical, roll and pitch motions are all considered. This is a nontrivial advancement compared with the recent work [10, 32]. Accommodation of unknown dynamics without using any function approximators can improve the computational efficiency and eliminate the effort required for the control parameter tuning. Furthermore, the suspension response (e.g. overshoot, convergence rate and ultimate displacement) can be strictly guaranteed, which directly contributes to the enhancement of operation safety for active suspension systems. This control design is model independent, and the derived control actions are with a proportional-like form. Hence, it is easy to implement, and may be more preferable in practice.

The contributions of this paper are summarized as follows:

a) This paper addresses active suspension control for full-car systems, where the nonlinear dynamics of springs and dampers in the systems are not necessarily known. Hence, the modeling efforts required for the control designs can be reduced.

b) An approximation-free control is obtained for active suspension by tailoring the idea of $[9,31]$. The predefined suspension response of vehicle motions is retained, while the function approximators are not used. Thus, the computational efficiency and system operation safety can be enhanced.

c) A dynamic simulator with realistic vehicle dynamics is built in a commercial vehicle simulation software Carsim 8.1. Comparative simulation results are given to exemplify the proposed control strategy and illustrate its superior responses.

The paper is structured as follows. The modeling of full-car suspension systems and preliminaries are given in Section II. Section III presents the AFC design and analysis of the closed-loop system stability. Simulation results are provided in Section IV and Section V gives some conclusions.

\section{Modelling OF Full-CAR AND PRELIMINARIES}

A. Modeling of Full-car Suspension System
In this paper, a nonlinear uncertain full-car model with four independent active suspension systems is considered, as shown in Fig.1. This full-car suspension system has seven degrees of freedom (DOFs), e.g. vertical, roll and pitch motions of the sprung mass, and the vertical motions of the four unsprung masses connected to the wheels (front-right, front-left, rear-right and rear-left) [10]. The variables of this suspension model as shown in Fig.1 and model (1) are defined as: $M$ is the sprung mass and $m_{i}, i=1 \ldots 4$ is the unsprung mass, representing the car body and the wheel assembly, respectively; $I_{\phi}$ and $I_{\theta}$ denote the mass moment of inertia for the roll and pitch motions. $F_{s i}$ and $F_{d i}$ are the forces produced by the springs and dampers. $k_{s i}$ and $k_{d i}$ are the spring stiffening coefficients and damper damping coefficients. $k_{t i}$ is the stiffness of the four wheels. $z_{s}, \phi$ and $\theta$ define the vertical, roll and pitch motions of the vehicle body. $z_{u i}$ and $y_{i}$ denote the displacement of the unsprung mass and road displacement input. $a, b, c$ and $d$ are the distances of the suspensions to the center of the vehicle body mass. The control inputs $u_{i}$ are the forces produced by the four active suspension systems to eliminate the effect of the road roughness. $V$ is the driving velocity of the car.

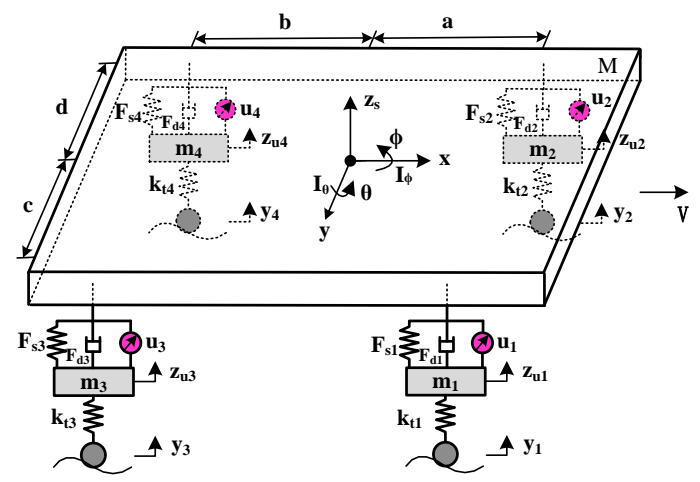

Fig.1 Schematic of full-car active suspension systems.

The detailed mathematical model of active suspension system shown in Fig.1 can be developed as [10, 32]:

$$
\left\{\begin{array}{l}
M \ddot{z}_{s}=-F_{s 1}-F_{s 2}-F_{s 3}-F_{s 4}-F_{d 1}-F_{d 2}-F_{d 3}-F_{d 4}+u_{z} \\
m_{1} \ddot{z}_{u 1}=F_{s 1}+F_{d 1}-k_{t 1}\left(z_{u 1}-y_{1}\right)-u_{1} \\
m_{2} \ddot{z}_{u 2}=F_{s 2}+F_{d 2}-k_{t 2}\left(z_{u 2}-y_{2}\right)-u_{2} \\
m_{3} \ddot{z}_{u 3}=F_{s 3}+F_{d 3}-k_{t 3}\left(z_{u 3}-y_{3}\right)-u_{3} \\
m_{4} \ddot{z}_{u 4}=F_{s 4}+F_{d 4}-k_{t 4}\left(z_{u 4}-y_{4}\right)-u_{4} \\
I_{\phi} \ddot{\phi}=c\left(F_{s 1}+F_{s 3}+F_{d 1}+F_{d 3}\right)-d\left(F_{s 2}+F_{s 4}+F_{d 2}+F_{d 4}\right)+u_{\phi} \\
I_{\theta} \ddot{\theta}=b\left(F_{s 3}+F_{s 4}+F_{d 3}+F_{d 4}\right)-a\left(F_{s 1}+F_{s 2}+F_{d 1}+F_{d 2}\right)+u_{\theta}
\end{array}\right.
$$

In (1), $u_{z}, u_{\phi}$ and $u_{\theta}$ denote the lumped control forces, which can be calculated in terms of the realistic control actions $u_{i}$. Hence, the formulation can be expressed as follows:

$$
\left\{\begin{array}{l}
u_{z}=u_{1}+u_{2}+u_{3}+u_{4} \\
u_{\phi}=d\left(u_{2}+u_{4}\right)-c\left(u_{1}+u_{3}\right) \\
u_{\theta}=a\left(u_{1}+u_{2}\right)-b\left(u_{3}+u_{4}\right)
\end{array}\right.
$$

Then based on (2) and the assumption $c u_{3}-d u_{4}=0$ used in 
$[10,32,34,35]$, the real control inputs $u_{i}, i=1 \ldots 4$ for active suspension systems can be calculated based on the derived three control actions $u_{z}, u_{\phi}$ and $u_{\theta}$ as:

$$
\left\{\begin{array}{l}
u_{1}=\left[b d u_{z}+d u_{\theta}-(a+b) u_{\phi}\right] /[(a+b)(c+d)] \\
u_{2}=\left[c b u_{z}+c u_{\theta}+(a+b) u_{\phi}\right] /[(a+b)(c+d)] \\
u_{3}=\left[d\left(a u_{z}-u_{\theta}\right)\right] /[(a+b)(c+d)] \\
u_{4}=\left[c\left(a u_{z}-u_{\theta}\right)\right] /[(a+b)(c+d)]
\end{array}\right.
$$

Hence, the objective is to design control for $u_{z}, u_{\phi}$ and $u_{\theta}$ based on (1), and then calculate the realistic control actions $u_{i}$ based on (3). It should be noted that the full-car suspension model described by (1) is used for the stability analysis only, and the exact force dynamics are not required for the control implementation. Nevertheless, the idea proposed in this paper can be extended to other suspension systems with different DOFs provided that a similar relationship as (3) can be found for force allocations.

To facilitate subsequent control designs, we first reformulate full-car system (1) as a state-space model. Define the system states as $x_{1}=z_{s}, x_{2}=\dot{z}_{s}, x_{3}=\phi, x_{4}=\dot{\phi}, x_{5}=\theta, x_{6}=\dot{\theta}, x_{7}=z_{u 1}$, $x_{8}=\dot{z}_{u 1}, x_{9}=z_{u 2}, x_{10}=\dot{z}_{u 2}, x_{11}=z_{u 3}, x_{12}=\dot{z}_{u 3}, x_{13}=z_{u 4}, x_{14}=\dot{z}_{u 4}$. Then the system (1) can be rewritten as:

$$
\left\{\begin{array}{l}
\dot{x}_{1}=x_{2} \\
\dot{x}_{2}=\frac{1}{M}\left(-F_{s 1}-F_{s 2}-F_{s 3}-F_{s 4}-F_{d 1}-F_{d 2}-F_{d 3}-F_{d 4}\right)+\frac{1}{M} u_{z} \\
\dot{x}_{3}=x_{4} \\
\dot{x}_{4}=\frac{1}{I_{\phi}}\left[c\left(F_{s 1}+F_{s 3}+F_{d 1}+F_{d 3}\right)-d\left(F_{s 2}+F_{s 4}+F_{d 2}+F_{d 4}\right)\right]+\frac{1}{I_{\phi}} u_{\phi} \\
\dot{x}_{5}=x_{6} \\
\dot{x}_{6}=\frac{1}{I_{\theta}}\left[b\left(F_{s 3}+F_{s 4}+F_{d 3}+F_{d 4}\right)-a\left(F_{s 1}+F_{s 2}+F_{d 1}+F_{d 2}\right)\right]+\frac{1}{I_{\theta}} u_{\theta}
\end{array}\right.
$$

$$
\left\{\begin{array}{l}
\dot{x}_{7}=x_{8} \\
\dot{x}_{8}=\frac{1}{m_{1}}\left[F_{s 1}+F_{d 1}-k_{t 1}\left(x_{7}-y_{1}\right)\right]-\frac{1}{m_{1}} u_{1} \\
\dot{x}_{9}=x_{10} \\
\dot{x}_{10}=\frac{1}{m_{2}}\left[F_{s 2}+F_{d 2}-k_{t 2}\left(x_{9}-y_{2}\right)\right]-\frac{1}{m_{2}} u_{2} \\
\dot{x}_{11}=x_{12} \\
\dot{x}_{12}=\frac{1}{m_{3}}\left[F_{s 3}+F_{d 3}-k_{t 3}\left(x_{11}-y_{3}\right)\right]-\frac{1}{m_{3}} u_{3} \\
\dot{x}_{13}=x_{14} \\
\dot{x}_{14}=\frac{1}{m_{4}}\left[F_{s 4}+F_{d 4}-k_{t 4}\left(x_{13}-y_{4}\right)\right]-\frac{1}{m_{4}} u_{4}
\end{array}\right.
$$

where the parameters $1 / M, 1 / I_{\phi}$ and $1 / I_{\theta}$ in (4) are bounded by positive constants $\theta_{1 \min } \ldots \theta_{3 \min }$ and $\theta_{1 \max } \ldots \theta_{3 \max }$ as $\theta_{1 \min } \leq 1 / M \leq \theta_{1 \max }, \theta_{2 \min } \leq 1 / I_{\phi} \leq \theta_{2 \max }$ and $\theta_{3 \min } \leq 1 / I_{\theta} \leq \theta_{3 \max }$.

In practical vehicle suspension systems, the applied forces are the functions of sprung/unsprung masses and tires motions.
Hence, the dynamics of $F_{s i}$ and $F_{d i}$ can be denoted [10, 32] as $F_{s i}=F_{s i}\left(x_{1} \ldots x_{6}, \bar{x}\right), F_{d i}=F_{d i}\left(x_{1} \ldots x_{6}, \bar{x}\right)$, where $\bar{x}=\left[x_{7} \ldots x_{14}\right]$. Therefore, the forces $F_{s i}$ and $F_{d i}$ are all bounded in a sufficient compact set in practice. In the proposed control, we do not require accurate system dynamics, e.g. dynamics of springs and dampers as required in $[10,32,34,35]$. Instead, only the vehicle motions $x_{1}, \ldots, x_{6}$ and wheel base (i.e. the distance between the wheels $a, b, c, d)$ are used in this paper, while the unknown forces and system dynamics are all handled.

Remark 1: In most of existing active suspension control designs, the forces of the springs and dampers in the suspension models (4) and (5) are usually assumed as linear functions, and/or their generation dynamics are fully known [10], which are stringent and unrealistic in practical applications. To address this issue, the unknown nonlinear dynamics generated by the springs and dampers are considered in this paper. More specifically, realistic forces embedded in Carsim are adopted in our case studies, whose generation models are unknown. This implies that the proposed control can cover more realistic applications, whilst requiring less information and reduced modeling effort in the control synthesis.

Remark 2: In the control design for systems with unknown nonlinearities and uncertainties, the unknown dynamics can be compensated by incorporating function approximators (e.g. NNs and FLSs) into the adaptive control implementation [20]. However, the function approximation based control methods usually have complex structures and impose demanding computational costs. Specifically, it is still a nontrivial task to tune large amount of parameters used in the adaptive control methods [20]. Moreover, the potentially sluggish transient response (online training of NNs and FLSs requires fairly long time to reach convergence) also limits the practical application. Remark 3: It is noted that the transient suspension response (e.g. convergence rate, overshoot) during the first few seconds is a critical issue, since too sluggish convergence or large overshoot may degrade the ride comfort, or even cause damage of suspension components. However, most of existing active control designs (e.g. [9-12, 21]) can merely guarantee the steady-state motion behaviors, i.e. $x_{j} \rightarrow 0, j=1,3,5$, but they cannot address the transient suspension behaviors explicitly.

Remark 4: It is noted that the realistic active suspension systems are operated by current or pressure applied to actuators (e.g. hydraulic or pneumatic actuators) to produce the required forces $u_{i}, i=1 \ldots 4$. Since this paper mainly focuses on presenting and validating a novel active suspensions control method to deal with uncertain dynamics and unknown nonlinearities without using function approximators, the actuator dynamics are not considered explicitly.

Inspired by the above discussions, this paper will propose control designs for obtaining $u_{z}, u_{\phi}$ and $u_{\theta}$ to accommodate the road excitations so as to maintain the vertical, roll and pitch motions $x_{j} \rightarrow 0, j=1,3,5$ (i.e. $z_{s}, \phi$ and $\theta$ ) of the vehicle body, while both the predefined transient and steady-state suspension responses can be retained without using function approximator. 


\section{B. Preliminaries}

To accomplish the stability analysis of the closed-loop control system, the following preliminaries of the initial value problem [33] are briefly introduced:

$$
\dot{\zeta}(t)=v(t, \zeta), \zeta(0)=\zeta^{0} \in \Omega_{\zeta}
$$

with $v: R_{+} \times \Omega_{\zeta} \rightarrow R^{n}$ being a continuous function and $\Omega_{\zeta} \in R^{n}$ is a nonempty open set.

Definition 1 [33]: The solution of the initial value problem (6) is maximal, if its solution $\zeta(t)$ has no proper right extension.

Theorem 1 [33]: For the initial value problem (6), if $v(t, \zeta)$ fulfills: a) locally Lipschitz on $\zeta(t)$ for $t>0$; b) piecewise continuous on $t$ for each $\zeta(t) \in \Omega_{\zeta}$; and c) locally integrable on $t$ for each $\zeta(t) \in \Omega_{\zeta}$. Then, there exists a unique maximal solution $\zeta(t)$ on the time interval $\left[0, \tau_{\max }\right)$ with $\tau_{\max } \in\left\{\mathbb{R}_{+}^{*}, \infty\right\}$ such that $\zeta(t) \in \Omega_{\zeta}$ for $\forall t \in\left[0, \tau_{\max }\right)$.

Proposition 1 [33]: If the conditions of Theorem 1 are true, then for a maximal solution $\zeta(t)$ on the time interval $\left[0, \tau_{\max }\right)$ with $\tau_{\max }<\infty$ and any compact set $\bar{\Omega}_{\zeta} \subset \Omega_{\zeta}$, there exists a time instant $t_{1} \in\left[0, \tau_{\max }\right)$ such that $\zeta\left(t_{1}\right) \notin \bar{\Omega}_{\zeta}$.

\section{ACtive SuSPENSION CONTROL AND ANALYSIS}

For completeness, we first briefly present the concept of the PPF and error transformation to characterize the suspension response of $x_{k}$ including the convergence rate and overshoot. Then, we will present the AFC schemes to regulate the vehicle motions $x_{k}$ with the predefined error constraints.

\section{A. Prescribed Performance Function}

To guarantee the suspension of vehicle motions $x_{j}$ within a predefined bounded region, we choose the following positive decreasing function $\varphi_{k}(t): R^{+} \rightarrow R^{+}$as the PPF [26-28]:

$$
\varphi_{k}(t)=\left(\varphi_{k 0}-\varphi_{k \infty}\right) e^{-a_{k} t}+\varphi_{k \infty}, \quad k=1, \ldots, 6
$$

where $\varphi_{k 0}>\varphi_{k \infty}>0$ and $a_{k}>0$ are positive constants set to predefine the initial error, ultimate error and convergence speed, respectively. Clearly, the following facts can be verified:

1) $\varphi_{k}(0)=\left(\varphi_{k 0}-\varphi_{k \infty}\right) e^{-a_{k} \cdot 0}+\varphi_{k \infty}=\varphi_{k 0}$;

2) $\lim _{t \rightarrow 0} \varphi_{k}(0) \rightarrow \varphi_{k 0}, \lim _{t \rightarrow+\infty} \varphi_{k}(t) \rightarrow \varphi_{k \infty}$.

Then we can use $\varphi_{k}(t)$ to construct predefined boundaries, within which the system states $x_{k}$ can be retained. This can be formulated as:

$$
-\delta \varphi_{k}(t)<x_{k}(t)<\delta \varphi_{k}(t), \forall t>0
$$

where $\delta$ is a positive constant chosen by the designers to fulfill the initial condition $\varphi_{k 0} \delta>\left|x_{k}(0)\right|$.

Remark 5: In the PPF formulation given in (7) and (8), $a_{k}$ represents the convergence rate; $\varphi_{k \infty}$ defines the ultimate steady-state error; $-\delta \varphi_{k}(0)$ and $\delta \varphi_{k}(0)$ account for the lower bound of the undershoot and upper bound of the overshoot [26, 28]. In this sense, both the transient and steady-state performance can be a priori designed by tuning the parameters $a_{k}, \delta, \varphi_{k 0}, \varphi_{k \infty}$.

\section{B. Error Transformation}

The key idea of PPF control is to represent the condition (8) into an equivalent "unconstrained" one as [26, 28] by introducing a coordinate transformation on the control error dynamics. For this purpose, we define $\zeta_{k} \in R$ as the normalized error and $S\left(\zeta_{k}\right) \in R$ is a smooth and strictly increasing function of $\zeta_{k}$, which satisfies the following conditions:
1) $-\delta<S\left(\zeta_{k}\right)<\delta, \forall \zeta_{k} \in L_{\infty}$
2) $\lim _{\zeta_{k} \rightarrow+\infty} S\left(\zeta_{k}\right)=\delta, \lim _{\zeta_{k} \rightarrow-\infty} S\left(\zeta_{k}\right)=-\delta$

From the properties of $S\left(\zeta_{k}\right)$, it can be verified that the condition (8) equals to:

$$
x_{k}(t)=\varphi_{k}(t) S\left(\zeta_{k}\right)
$$

Hence, one can use the function $S\left(\zeta_{k}\right)$ given by:

$$
S\left(\zeta_{k}\right)=\frac{\delta e^{\zeta_{k}}-\delta e^{-\zeta_{k}}}{e^{\zeta_{k}}+e^{-\zeta_{k}}}
$$

Based on the facts that $\varphi_{k 0}>\varphi_{k \infty}>0$ and $S\left(\zeta_{k}\right)$ is strictly increasing, we can calculate its inverse function as:

$$
\varepsilon_{k}=S^{-1}\left[\zeta_{k}\right]=\frac{1}{2} \ln \left(\frac{\delta+\zeta_{k}}{\delta-\zeta_{k}}\right)
$$

which defines a mapping from the unconstrained error $x_{k}$ to the intermediate variable $\zeta_{k}$ with the transformation (9). Then, the stabilization of the transformed error $\varepsilon_{k}$ is sufficient to guarantee the suspension of vehicle motions $x_{k}$ given in (4) with the prescribed bound given in (8). In this sense, as stated in $[26,28]$, the tracking control with a predefined error bound (8) can be reduced to retain the boundedness of the transformed error (11). Hence, in the next section, the transformed variables (11) will be used in the AFC design.

\section{AFC Controller Design for Vertical Motion}

The control design objective is to regulate the vertical, roll and pitch motions $x_{1}, x_{3}, x_{5}$ (i.e. $z_{s}, \phi$ and $\theta$ ) governed by (4) into the predefined boundary (8). For this purpose, we will present a new control design, where the PPF (7) and error transformation (11) will be utilized throughout the following developments and analyses.

We first present the control design for vertical motion $x_{1}$, while the controllers for the roll and pitch motions $x_{3}, x_{5}$ can be obtained similarly.

Step 1: For the vertical motion dynamics given in (4), we first define the suspension error as $x_{1}$, and then the normalized error is derived as $\zeta_{1}(t)=x_{1}(t) / \varphi_{1}(t)$ with a PPF defined in (7) as:

$$
\varphi_{1}(t)=\left(\varphi_{10}-\varphi_{1 \infty}\right) e^{-a_{1} t}+\varphi_{1 \infty}
$$

where $\varphi_{10}, \varphi_{1 \infty}$ and $a_{1}$ are set as positive constants such that the initial condition $\left|x_{1}(0)\right|<\delta \varphi_{10}$ is fulfilled.

Then, one can construct the first virtual control as:

$$
u_{1}=-k_{1} \varepsilon_{1}=-\frac{k_{1}}{2} \ln \left(\frac{\delta+\zeta_{1}}{\delta-\zeta_{1}}\right)
$$

where $k_{1}>0$ is a control gain, and the transformed error is derived along with (11) as: 


$$
\varepsilon_{1}=S^{-1}\left[\zeta_{1}\right]=\frac{1}{2} \ln \left(\frac{\delta+\zeta_{1}}{\delta-\zeta_{1}}\right)
$$

Step 2: By using the obtained virtual control action, one can denote the virtual control error as:

$$
e_{1}(t)=x_{2}(t)-u_{1}(t)
$$

Then the corresponding nominalized virtual control error is given by $\zeta_{2}(t)=e_{1}(t) / \varphi_{2}(t)$, where the second $\operatorname{PPF} \varphi_{2}(t)$ is given as:

$$
\varphi_{2}(t)=\left(\varphi_{20}-\varphi_{2 \infty}\right) e^{-a_{2} t}+\varphi_{2 \infty}
$$

with $\varphi_{20}, \varphi_{2 \infty}$ and $a_{2}$ being properly selected positive constants to guarantee the initial condition $\left|e_{1}(0)\right|<\delta \varphi_{20}$.

Then, the required control action $u_{z}$ for the vertical motion can be described by:

$$
u_{z}=-k_{2} \varepsilon_{2}=-\frac{k_{2}}{2} \ln \left(\frac{\delta+\zeta_{2}}{\delta-\zeta_{2}}\right)
$$

with $k_{2}>0$ being a constant control gain, and the transformed error $\varepsilon_{2}$ of $\zeta_{2}(t)$ is calculated by:

$$
\varepsilon_{2}=S^{-1}\left[\zeta_{2}\right]=\frac{1}{2} \ln \left(\frac{\delta+\zeta_{2}}{\delta-\zeta_{2}}\right)
$$

\section{AFC Controller Design for Roll and Pitch Motions}

The control actions $u_{\phi}$ and $u_{\theta}$ for the roll and pitch motions $x_{3}$ and $x_{5}$ can be designed following similar manipulations as that for vertical motion $x_{1}$, which can be briefly given as: 1) The control action $u_{\phi}$ of roll motion $x_{3}$ is designed as:

$$
\begin{aligned}
& u_{\phi}=-k_{4} \varepsilon_{4}=-\frac{k_{4}}{2} \ln \left(\frac{\delta+\zeta_{4}}{\delta-\zeta_{4}}\right) \\
& \zeta_{4}(t)=e_{2}(t) / \varphi_{4}(t) \\
& e_{2}(t)=x_{4}(t)-u_{3}(t), \\
& u_{3}=-k_{3} \varepsilon_{3}=-\frac{k_{3}}{2} \ln \left(\frac{\delta+\zeta_{3}}{\delta-\zeta_{3}}\right) \\
& \zeta_{3}(t)=x_{3}(t) / \varphi_{3}(t)
\end{aligned}
$$

where $k_{3}, k_{4}>0$ are positive control gains, $\varphi_{3}(t)$ and $\varphi_{4}(t)$ are PPFs defined in (7) with $\varphi_{30}, \varphi_{3 \infty}, \alpha_{3}$ and $\varphi_{40}, \varphi_{4 \infty}, \alpha_{4}$ being properly selected positive constants to fulfill the initial conditions $\left|x_{3}(0)\right|<\delta \varphi_{30}$ and $\left|e_{2}(0)\right|<\delta \varphi_{40}$.

2) The control action $u_{\theta}$ of the pitch motion $x_{5}$ is designed as:

$$
\begin{aligned}
& u_{\theta}=-k_{6} \varepsilon_{6}=-\frac{k_{6}}{2} \ln \left(\frac{\delta+\zeta_{6}}{\delta-\zeta_{6}}\right) \\
& \zeta_{6}(t)=e_{3}(t) / \varphi_{6}(t) \\
& e_{3}(t)=x_{6}(t)-u_{5}(t) \\
& u_{5}=-k_{5} \varepsilon_{5}=-\frac{k_{5}}{2} \ln \left(\frac{\delta+\zeta_{5}}{\delta-\zeta_{5}}\right) \\
& \zeta_{5}(t)=x_{5}(t) / \varphi_{5}(t)
\end{aligned}
$$

where $k_{5}, k_{6}>0$ are positive control gains, the PPFs $\varphi_{5}(t)$ and $\varphi_{6}(t)$ are defined in (7) with properly selected positive constants $\varphi_{50}, \varphi_{5 \infty}, \alpha_{5}$ and $\varphi_{60}, \varphi_{6 \infty}, \alpha_{6}$ such that the initial conditions $\left|x_{5}(0)\right|<\delta \varphi_{50}$ and $\left|e_{3}(0)\right|<\delta \varphi_{60}$ are fulfilled.

Hence, as explained in (3), we can calculate the realistic control inputs $u_{i}, i=1,2,3,4$ applied on the practical suspension system as long as the control actions $u_{z}, u_{\phi}$ and $u_{\theta}$ are obtained by using (17), (19) and (20).

Remark 6: It is noted in the above control designs that the intermediate control laws $u_{1}, u_{3}, u_{5}$ are similar to the virtual control variables in the backstepping $[10,16]$. However, unlike backstepping methods where the derivatives of the virtual controls needs to be obtained, the proposed AFC actions (17), (19) and (20) are with a proportional-like form of the transform errors $\varepsilon_{k}=S^{-1}\left[\zeta_{k}\right]$ for the normalized errors $\zeta_{k}$. Clearly, the proposed controller has a simpler structure, making its practical implementation easier. Moreover, the 'explosion of complexity' issue encountered in the backstepping methods is also avoided. Remark 7: As shown in (17), (19) and (20), the AFC does not require any function approximators, while the unknown dynamics in the suspension system (4) can be accommodated effectively. Hence, this control has reduced computational costs, and avoids the sluggish online learning procedure. This is clearly different to the well-known function approximation based adaptive control methods, where NNs or FLSs are used to estimate and compensate the unknown nonlinearities.

Remark 8: In the proposed AFC method, the parameters to be tuned can be classified into two categories, i.e. PPF parameters $\varphi_{k 0}, \varphi_{k \infty}, a_{k}, \delta$ and control gains $k_{k}$. The parameter tuning of the AFC is more straightforward than adaptive control schemes and thus easy to implement for practitioners. The required initial conditions $\left|x_{1}(0)\right|<\delta \varphi_{10},\left|e_{1}(0)\right|<\delta \varphi_{20},\left|x_{3}(0)\right|<\delta \varphi_{30}$, $\left|e_{2}(0)\right|<\delta \varphi_{40},\left|x_{5}(0)\right|<\delta \varphi_{50},\left|e_{3}(0)\right|<\delta \varphi_{60}$ can be satisfied by choosing large PPF parameters $\varphi_{k 0}$ and $\delta$. The convergence rate $a_{k}$ can be set small initially and then adjusted large to achieve fast error convergence. The ultimate error bounds $\varphi_{k \infty}$ can be set large in the initial phase and then reduced to obtain smaller errors. On the other hand, the control gains $k_{k}$ are related to the control error and the smoothness and amplitude of control actions, thus they can be set small initially and then increased gradually to seek for a tradeoff between the control response and the required control actions.

The practical implementation of the proposed control method can be described as the following algorithm:

Algorithm: Online Implementation of the proposed AFC
1: Initialize the PPF parameters $\varphi_{k}, k=1, \ldots, 6$ for vertical,
roll and pitch motions;
2: Calculate the suspension errors $e_{k}(t)$ according to the
measured variables $x_{1}, \ldots, x_{6}$;
3: Define the normalized errors $\zeta_{k}$ as:
\[ \zeta_{k}=e_{k}(t) / \varphi_{k} \]
where $\varphi_{k}$ is the PPF defined in $(7)$.
4: Calculate the transformed errors $\varepsilon_{k}$ as:
\[ \varepsilon_{k}=S^{-1}\left[\zeta_{k}\right]=\frac{1}{2} \ln \left(\frac{\delta+\zeta_{k}}{\delta-\zeta_{k}}\right) \] 
5: Design the control signals as:

$$
u_{k}=-k_{k} \varepsilon_{k}=-\frac{k_{k}}{2} \ln \left(\frac{\delta+\zeta_{k}}{\delta-\zeta_{k}}\right)
$$

6: Allocate the control signals $u_{z}=-k_{2} \varepsilon_{2}, u_{\phi}=-k_{4} \varepsilon_{4}$, $u_{\theta}=-k_{6} \varepsilon_{6}$ based on (3) and obtain the real control actions $u_{1}, \ldots, u_{4}$ to be applied on the system.

7: Go back to step 2 .

\section{E. Stability and Convergence Analysis}

The stability of the closed-loop control system and the convergence of suspension displacements will be examined in this section. As shown in (4), the vertical, roll and pitch motions $z_{s}, \phi$ and $\theta$ and the proposed corresponding controllers have similar structures. Hence, we will provide detailed analysis for the vertical motion $x_{1}, x_{2}$ only. Similar analysis can be conducted for the roll motion $x_{3}, x_{4}$ and pitch motion $x_{5}, x_{6}$, which will not be detailed due to the page limit.

Before presenting the convergence property of the proposed control system, we first derive the dynamics of the adopted transformed errors. Consider the definition of virtual control errors and the corresponding normalized errors $\zeta_{k}(t)$, one can obtain from (13)-(20):

$$
\begin{aligned}
& x_{1}=\zeta_{1} \varphi_{1}, x_{2}=\zeta_{2} \varphi_{2}+u_{1}, \\
& x_{3}=\zeta_{3} \varphi_{3}, x_{4}=\zeta_{4} \varphi_{4}+u_{3}, \\
& x_{5}=\zeta_{5} \varphi_{5}, x_{6}=\zeta_{6} \varphi_{6}+u_{5} .
\end{aligned}
$$

By using (24) and the explanations below (4), the governing dynamics of $F_{s i}$ and $F_{d i}$ in the suspension system (4) can be reformulated as:

$$
\begin{aligned}
& F_{s i}\left(x_{1} \ldots x_{6}, \bar{x}\right)=F_{s i}\left(\zeta_{1} \varphi_{1}, \zeta_{2} \varphi_{2}+u_{1}, \zeta_{3} \varphi_{3}, \zeta_{4} \varphi_{4}+u_{3}, \zeta_{5} \varphi_{5}, \zeta_{6} \varphi_{6}+u_{5}, \bar{x}\right) \\
& F_{d i}\left(x_{1} \ldots x_{6}, \bar{x}\right)=F_{d i}\left(\zeta_{1} \varphi_{1}, \zeta_{2} \varphi_{2}+u_{1}, \zeta_{3} \varphi_{3}, \zeta_{4} \varphi_{4}+u_{3}, \zeta_{5} \varphi_{5}, \zeta_{6} \varphi_{6}+u_{5}, \bar{x}\right)
\end{aligned}
$$

Based on the definition of the normalized errors $\zeta_{1}, \zeta_{2}$, their derivatives are calculated along (24) and (13)-(17) with (4) as:

$$
\begin{aligned}
\dot{\zeta}_{1}= & \frac{d\left(x_{1} / \varphi_{1}\right)}{d t}=\frac{1}{\varphi_{1}}\left(\dot{x}_{1}-\zeta_{1} \dot{\varphi}_{1}\right)=\frac{1}{\varphi_{1}}\left(\zeta_{2} \varphi_{2}+u_{1}-\zeta_{1} \dot{\varphi}_{1}\right) \\
= & v_{1}\left(t, \zeta_{1}, \zeta_{2}\right) \\
\dot{\zeta}_{2}= & \frac{d\left(e_{2} / \varphi_{2}\right)}{d t}=\frac{1}{\varphi_{2}}\left(\dot{e}_{2}-\zeta_{2} \dot{\varphi}_{2}\right)=\frac{1}{\varphi_{2}}\left(\dot{x}_{2}-\dot{u}_{1}-\zeta_{2} \dot{\varphi}_{2}\right) \\
= & \frac{1}{\varphi_{2}}\left[\frac { 1 } { M } \left(-\sum_{i=1}^{4} F_{s i}\left(\zeta_{1} \varphi_{1}, \zeta_{2} \varphi_{2}+u_{1}, \zeta_{3} \varphi_{3}, \zeta_{4} \varphi_{4}+u_{3}, \zeta_{5} \varphi_{5},\right.\right.\right. \\
& \left.\zeta_{6} \varphi_{6}+u_{5}, \bar{x}\right)-\sum_{i=1}^{4} F_{d i}\left(\zeta_{1} \varphi_{1}, \zeta_{2} \varphi_{2}+u_{1}, \zeta_{3} \varphi_{3}, \zeta_{4} \varphi_{4}+u_{3},\right. \\
& \left.\left.\left.\zeta_{5} \varphi_{5}, \zeta_{6} \varphi_{6}+u_{5}, \bar{x}\right)+u_{z}\right)-\dot{u}_{1}-\zeta_{2} \dot{\varphi}_{2}\right] \\
= & v_{2}\left(t, \zeta_{1}, \zeta_{2}, \zeta_{3}, \zeta_{4}, \zeta_{5}, \zeta_{6}\right)
\end{aligned}
$$

From (26)-(27), the dynamics of the normalized error vector $\zeta=\left[\zeta_{1}, \zeta_{2}, \zeta_{3}, \zeta_{4}, \zeta_{5}, \zeta_{6}\right]^{T}$ are represented in a compact form:

$$
\dot{\zeta}(t)=v(t, \zeta)=\left[\begin{array}{l}
v_{1}\left(t, \zeta_{1}, \zeta_{2}\right) \\
v_{2}\left(t, \zeta_{1}, \zeta_{2}, \zeta_{3}, \zeta_{4}, \zeta_{5}, \zeta_{6}\right)
\end{array}\right]
$$

Since the induced forces in (4) are assumed to be continuous functions with respect to time and its coordinates, the function $v(t, \zeta)$ given in (28) is piecewise continuous on time $t$, locally Lipschitz on $\zeta$ within a nonempty set $\Omega_{\zeta}=(-\delta, \delta) \times(-\delta, \delta)$.

Hence, the main results of this paper can be given as:

Theorem 2: For active suspension system (4), consider the AFC (17) with (13) is designed for the vertical motion $x_{1}$ under the initial conditions $\left|e_{k}(0)\right|<\varphi_{k 0}$, then all signals in the vertical dynamic system are bounded, and $x_{1}$ is retained within the prescribed bound defined in (8).

Proof: The proof is shown in the Appendix

It is noted that similar analysis results can also be claimed for the roll motion $x_{3}$ and pitch motion $x_{5}$, which implies that the suspension responses of $x_{3}$ and $x_{5}$ can be retained within the bound $-\delta \varphi_{3}(t)<x_{3}(t)<\delta \varphi_{3}(t),-\delta \varphi_{5}(t)<x_{5}(t)<\delta \varphi_{5}(t)$.

\section{Simulations}

In this section, numerical simulations with two different road profiles are carried out to validate the effectiveness of the proposed AFC method. To cover more realistic vehicle dynamics, we have built a dynamic simulator by using professional vehicle simulation software $\operatorname{Carsim}^{\circledR}$ and Matlab ${ }^{\circledR}$. For the purpose of demonstration, an E-SUV type vehicle model embedded in $\mathrm{Carsim}^{\circledR}$ is used. The full-car suspension dynamics are generated by Carsim $^{\circledR}$ based on the embedded realistic vehicle data, which are all unknown and thus not required in the control implementation, and the proposed AFC method is implemented in Simulink/Matlab. The control signals and the suspension system states are communicated between these two softwares in real time. The structure of the developed simulator can be found in Fig. 2.

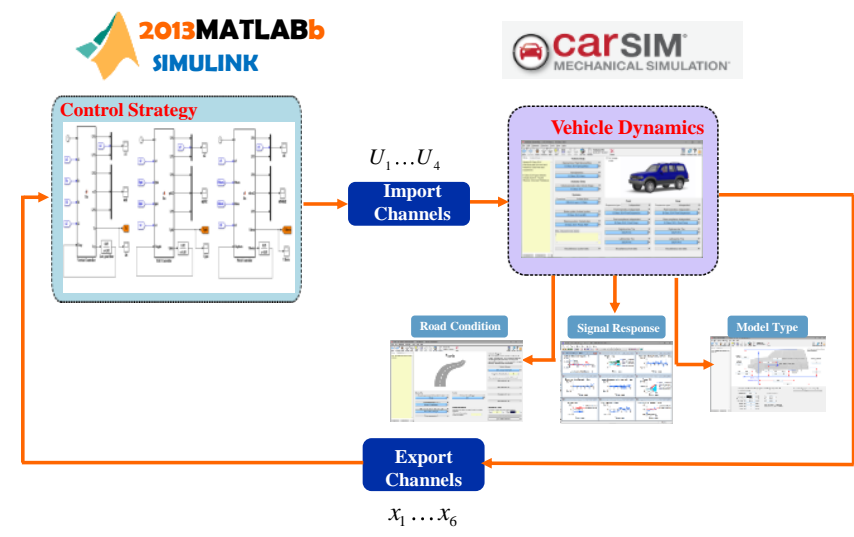

Fig.2 Diagram of the closed-loop dynamic simulator.

It is noted that in this developed simulator, the dynamics of full-car suspension system (1) are generated from Carsim ${ }^{\circledR}$ and their accurate models are unknown. Only the system states $x_{1}, x_{2}, \cdots, x_{6}$ that can be measured by using the configured sensors are required in this paper. In this sense, the plant to be controlled can be regarded as a "black box". This requirement is clearly less stringent than most of existing active suspension control results, which assume fully known suspension system dynamics. Hence, this control method can reduce the modeling effort as it is a model-independent control structure. 
The major parameters of the studied E-SUV type vehicle are listed in TABLE I, which are taken from Carsim $^{\circledR}$. It is worth mentioning that only parts of these parameters are used in the AFC strategy in comparison to the backstepping control (BSC) $[10,16]$, i.e. only the distances between the wheels $a, b, c, d$ are used to allocate control signals in (3). For comparison, passive suspension scheme embedded in the Carsim ${ }^{\circledR}$ is also tested, where the corresponding parameters, i.e., stiffness coefficients of spring $k_{s i}(i=1, \ldots, 4)$ and damper $k_{d i}(i=1, \ldots, 4)$, are also listed in Table I.

TABLE I PARAMETERS FOR E-SUV TYPE CAR MODEL

\begin{tabular}{cccc}
\hline \hline Symbol & Value & Symbol & Value \\
\hline$M$ & $1590 \mathrm{~kg}$ & $c$ & $0.7875 \mathrm{~m}$ \\
$I_{\phi}$ & $894.4 \mathrm{kgm}^{2}$ & $d$ & $0.7875 \mathrm{~m}$ \\
$I_{\theta}$ & $2687.1 \mathrm{kgm}^{2}$ & $V$ & $40 \mathrm{~km} / \mathrm{h}$ \\
$m_{i}$ & $120 \mathrm{~kg}$ & $k_{s i}$ & $46 \mathrm{~N} / \mathrm{mm}$ \\
$a$ & $1.18 \mathrm{~m}$ & $k_{d i}$ & $30000 \mathrm{Ns} / \mathrm{m}$ \\
$b$ & $1.77 \mathrm{~m}$ & & \\
\hline \hline
\end{tabular}

In the following, two driving road profiles embedded in Carsim $^{\circledR}$ are chosen to verify the active suspension system.

Scenario 1 (Bump road with example roughness): Unlike most of existing suspension results that only use bump road condition to test the effectiveness of active suspension control system, this paper incorporates the example road roughness into a bump road to conduct a more realistic test driving road condition ( $3.5 \mathrm{~cm}$ high and $40 \mathrm{~cm}$ long). The generated road profile is shown in Fig.3.

In the simulations, the initial suspension system states are $x_{k}(0)=0, k=1, \ldots, 14$. Following the aforementioned parameter tuning guidelines in Remark 8, the PPFs' parameters are set as: $\varphi_{1}(t)=(0.1-0.04) e^{-10 t}+0.04, \varphi_{2}(t)=(10000-6000) e^{-10 t}+4000$, $\varphi_{3}(t)=(5-2) e^{-15 t}+2, \varphi_{4}(t)=(5000-3000) e^{-20 t}+3000, \varphi_{5}(t)$ $=(6-3) e^{-22 t}+3$ and $\varphi_{6}(t)=(10000-6500) e^{-20 t}+6500$. The feedback control gains are set as $k_{1}=7000, k_{2}=9000, k_{3}=$ $7500, k_{4}=12000, k_{5}=5100$ and $k_{6}=7400$. Simulation results are given in Figs.4-5. In Fig.4, the responses of displacements and accelerations of the vehicle motions in the vertical, roll and pitch directions are provided. One can find from Fig.4 that the proposed active suspension control system has lower peaks and less fluctuations for both the vehicle motion displacements and accelerations in comparison to the passive suspension. This fact indicates that the proposed AFC method can isolate the vibrations transmitted to the vehicle body from irregular road roughness effectively to maintain the stability of vehicle body, which contributes to improving the ride comfort. The profiles of suspension deflections for four active suspension systems are shown in Fig.5, where all the deflections are within the allowable range $0.1 \mathrm{~m}$, which help to retain the driving safety. It is noted that there are inherent conflicts between the ride comfort and suspension deflections, which means that better ride comfort may result in larger suspension deflection. In the control design presented in this paper, we choose the ride comfort as the primary target. Hence, the suspension deflection may be larger than the passive scheme as shown in Fig.5. Moreover, there are significant couplings between the vertical, pitch and roll dynamics, which lead to asymmetric behaviors in the deflections as shown in Fig.5, which may also stem from the asymmetric forces applied on the left and right tires.

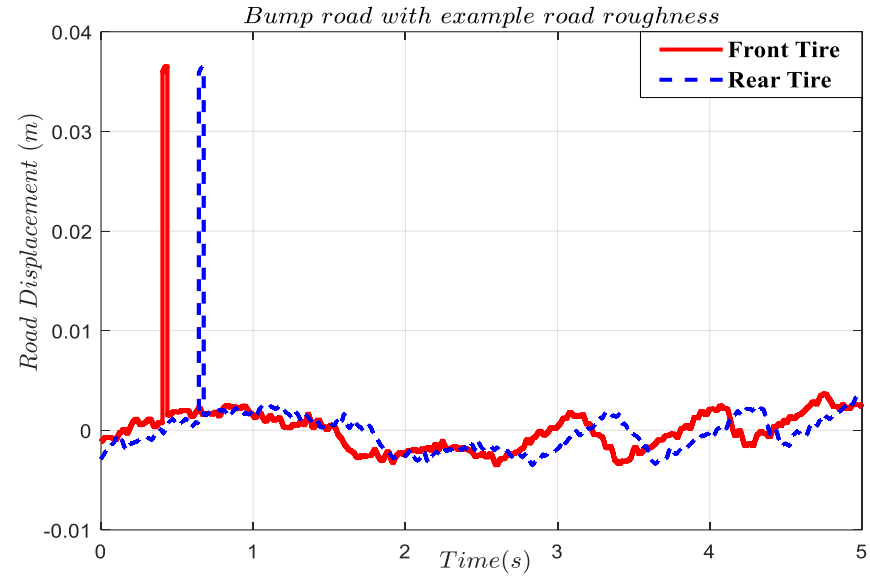

Fig.3 Ground road elevations of the combined bump road.

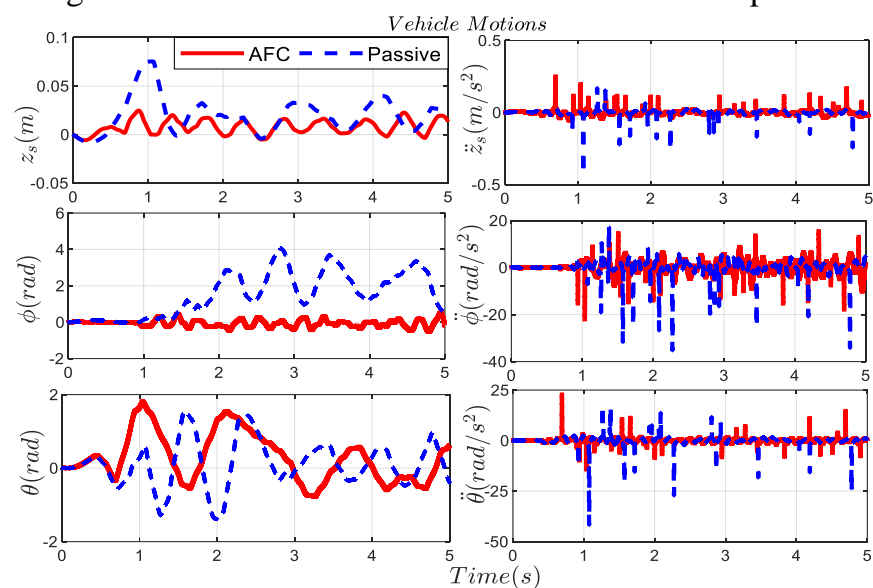

Fig.4 Vehicle motions and accelerations under bump road.

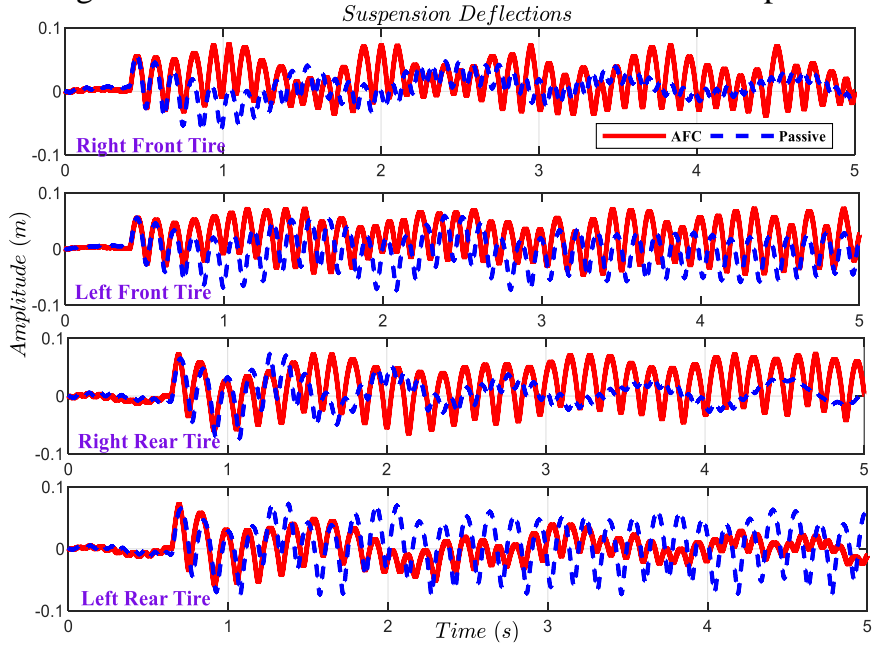

Fig.5 Profiles of suspension deflections.

Scenario 2 (Bounce sine sweep road): To further validate the efficiency and robustness of the AFC method, a more oscillated road condition, bounce sine sweep road, is selected, which is given in Fig.6. Considering the fact that the bounce sine sweep road is more aggressive (i.e. with fast varying frequency and 
amplitude) than bump road, the corresponding PPF boundaries can be further modified to enhance the control response as: $\varphi_{1}(t)=(1.2-0.5) e^{-2.5 t}+0.5, \varphi_{2}(t)=(8000-5000) e^{15 t}+5000$, $\varphi_{3}(t)=(15-10) e^{-3 t}+10, \varphi_{4}(t)=(8000-5000) e^{-1.5 t}+5000$, $\varphi_{5}(t)=(15-10) e^{-2.5 t}+10$ and $\varphi_{6}(t)=(8000-6000) e^{-2 t}+6000$. The feedback control gains are set as $k_{1}=4000, k_{2}=7880$, $k_{3}=1700, k_{4}=4830, k_{5}=1482$ and $k_{6}=4790$.

Figs.7-10 give comparative simulation results. It is shown in Fig.7 that the introduced AFC scheme achieves fairly good suspension performance under the bounce sine sweep road condition compared with the BSC method and passive suspension. Specifically, the vibrations of the vehicle body in the vertical, roll and pitch directions are significantly mitigated with the AFC method, and both the transient and steady-state suspension errors are the smallest among the three tested controllers. To evaluate the ride comfort of different suspension schemes, the acceleration signals that have been well recognized as a ride comfort performance index are also depicted in Fig.8. One can find from Fig.8 that both the AFC method and BSC method can reduce the amplitude of the accelerations of the vehicle body in the vertical, roll and pitch directions compared with passive suspension. In particular, the proposed AFC yields lower peaks than the BSC method. This fact indicates that the AFC scheme provides improved ride comfort over the BSC method.

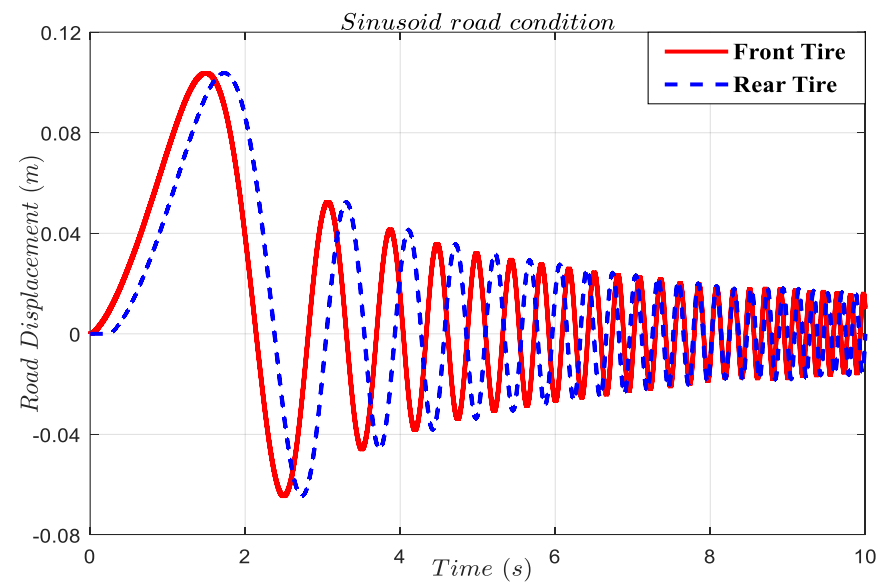

Fig.6 Ground road elevations of sinusoid road.

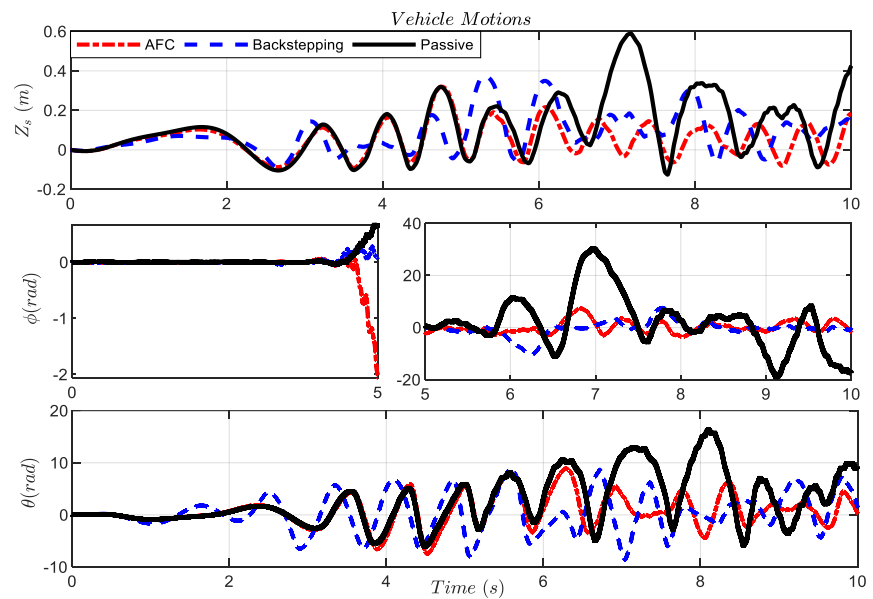

Fig.7 Vehicle motions under sinusoid road condition.
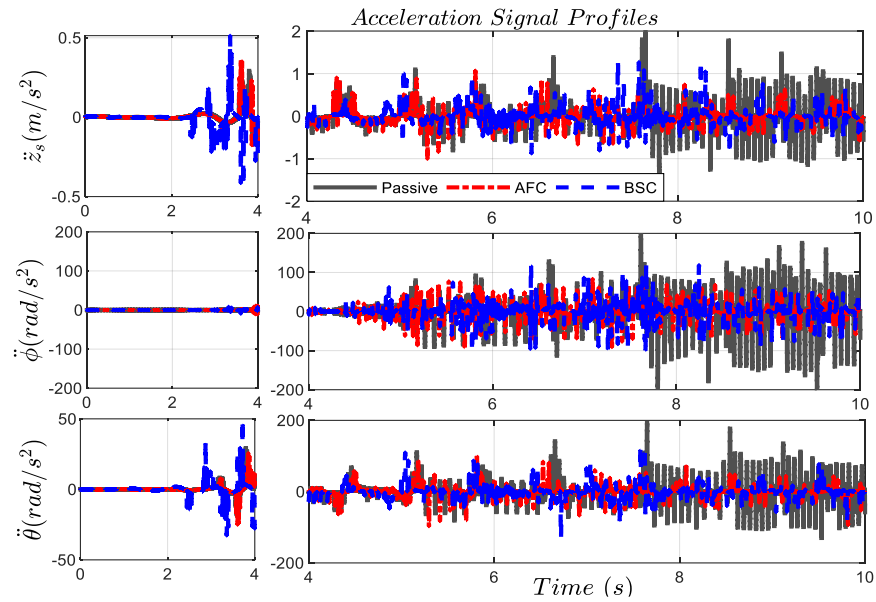

Fig. 8 Acceleration responses of vehicle motions.

To further quantitatively exemplify the performance of the proposed AFC method, three commonly used performance indices: Integral Absolute Error (IAE), Root Mean Square (RMS) and Maximum (MAX), are calculated for the three different controllers. Comparative results for the three control strategies under the sine sweep road condition are shown in Fig.9. From Fig.9, it can be clearly found that the proposed AFC method yields the smallest index values among the three given performance indices, which means that the AFC provides superior performs over the BSC method and passive suspension in terms of suspension motion behaviors. This again implies that the AFC has better capability to isolate the vehicle motions as much as possible from the road-induced shocks.

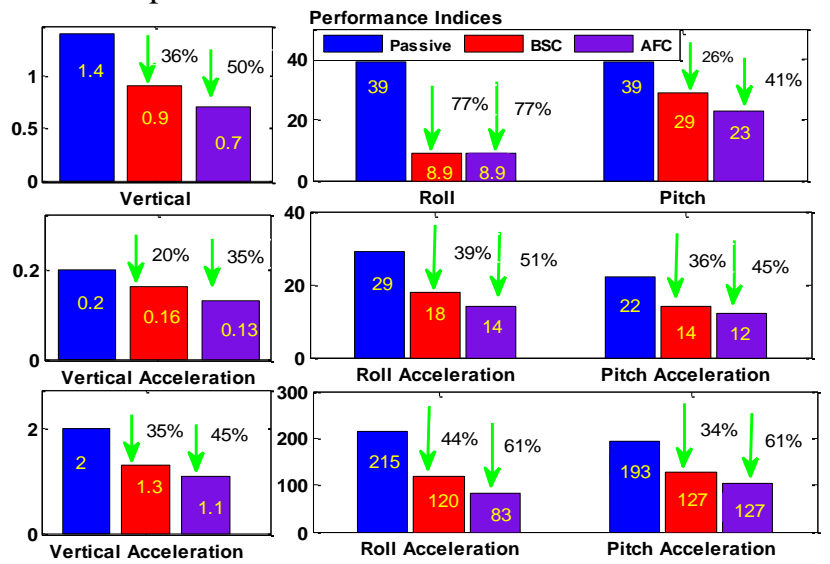

Fig.9 Performance evaluation of different methods.

Moreover, since the suspension strokes have certain effects on the driving safety, it must be limited within a resaonalbe bound especially in the presense of aggressive riding road conditon. Simulation results of the suspension deflections with sine sweep road excitation can be observed from Fig.10. As shown in Fig.10, the suspension stroke responses with both the AFC and BSC appraoches are all guaranteed within the allowable bounded set. Furthermore, the proposed AFC again can obtain less fluctuationss and lower peaks than BSC method and passive suspension, in particular in the high-frequency regimes. Comparative results of control inputs with the proposed AFC method and the BSC method are plotted in Fig.11. One may find from Fig.11 that although the control inputs of both methods are bounded, the AFC requires less forces and provides smoother control signals (i.e. less 
fluctuations) compared to the BSC method, i.e. it requires less control power due to the use of the prescribed performance function that leads to better suspension responses. This advantage is preferable in practical suspension system designs.

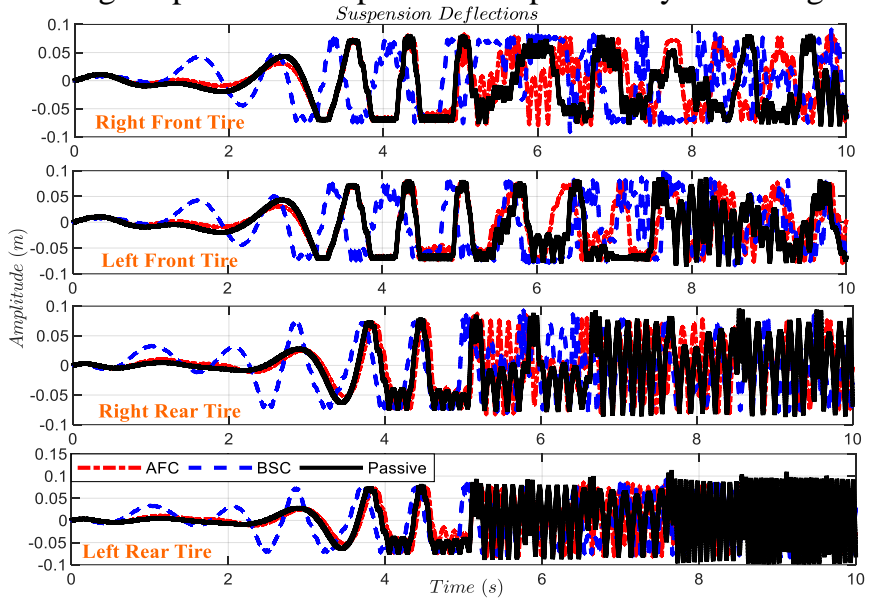

Fig.10 Comparative results of suspension deflections.
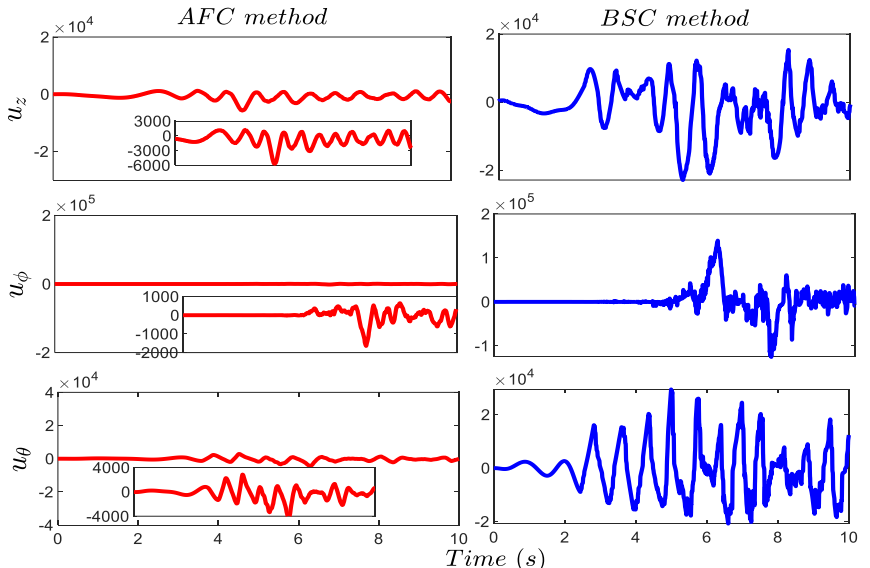

Fig.11 Control inputs of the proposed AFC and BSC method.

Moreover, to illustrate the computational efficiency of the proposed method, the computational time of simulations for the three control methods under two different road conditions (Scenario 1 and Scenario 2) is provided in Table II. One can find from Table II that the computational time of the AFC is dramatically decreased for both road conditions compared with adaptive control given in [36] and the BSC method. This is reasonable since the online training of the NNs weights involved in the adaptive control and the repeated calculation of the derivatives of virtual control actions in the BSC method are all avoided in the proposed AFC method.

Table II COMPARATIVE RESULTS OF COMPUTATIONAL TIME (SCENARIO 1/SCENARIO 2)

\begin{tabular}{lcc}
\hline \hline Methods & $\begin{array}{c}\text { Computational } \\
\text { time }(\mathrm{s})\end{array}$ & Decreasing ratio \\
\hline Adaptive control & $15.83 \mathrm{~s} / 19.1 \mathrm{~s}$ & Benchmark \\
BSC method & $10.76 \mathrm{~s} / 11.5 \mathrm{~s}$ & $68.1 \% / 66.1 \% \downarrow$ \\
AFC method & $4.46 \mathrm{~s} / 4.3 \mathrm{~s}$ & $71.8 \% / 77.5 \% \downarrow$ \\
\hline
\end{tabular}

Finally, to demonstrate the effectiveness of the proposed AFC method and the generality of using same control parameters under different road conditions, an extra simulation is carried out, where the simulation parameters used in Scenario 2 are also adopted for Scenario 1 since the required initial conditions can be fulfilled for both road conditions. It can be found from Fig.12 that both the transient and steady-state convergence can be retained for both road conditions, though the well-tuned parameters can contribute to better control performance as shown in the above simulations.

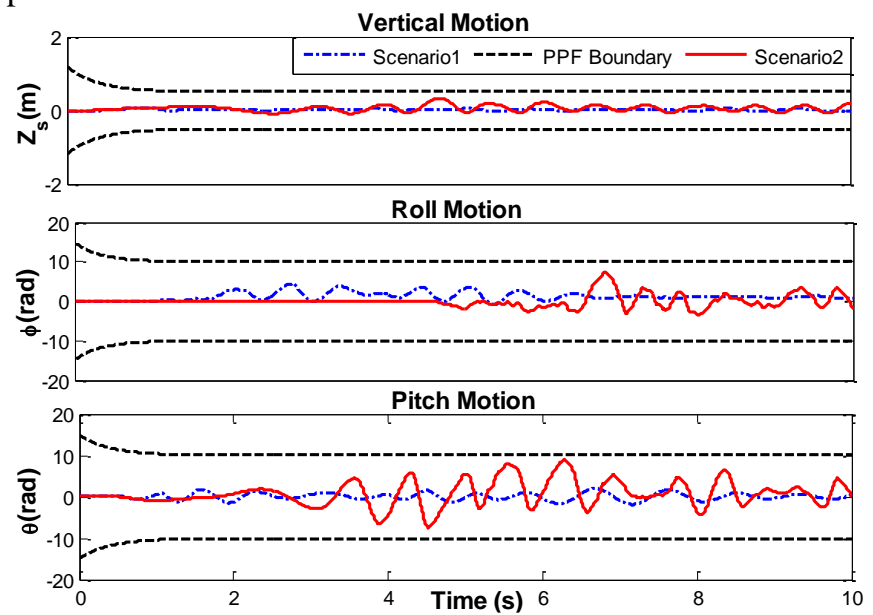

Fig.12 Control performance with same parameters for different road conditions.

To justify the implementation of the AFC in practical applications, the required variables of the proposed AFC method and the BSC method are summarized in Table III. For the BSC method, the vehicle forces $F_{d i}, F_{s i}$, inertial variables $M, I_{\phi}, I_{\theta}$, vehicle motions $x_{1}, \ldots, x_{6}$ and wheel base (i.e. the distance between the wheels $a, b, c, d)$ are all required to be measureable and available, while for the proposed AFC, only the vehicle motions and wheel base are required in the control implementation. In this respect, the proposed AFC may be more suited for practical active suspension systems due to its reduced modeling effort and less sensors.

TABLE III REQUIRED VARIABLES FOR TWO CONTROLLERS

\begin{tabular}{ccc}
\hline \hline & AFC Method & BSC Method \\
\hline Forces & - & $F_{d i}, F_{s i}$ \\
Inertial Variables & - & $M, I_{\phi}, I_{\theta}$ \\
Vehicle Motions & $x_{i}, i=1, \ldots, 6$ & $x_{i}, i=1, \ldots, 6$ \\
Wheel Base & $a, b, c, d$ & $a, b, c, d$ \\
\hline
\end{tabular}

It should be noted that the control designs for the vertical, pitch and roll motions of the full-car suspension system presented in this paper have been derived separately as shown in (17), (19) and (20), and then the obtained control actions are allocated based on (3). The motion dynamics of each DOF of the sprung mass are indeed the same as those of quarter-car suspension systems studied in the literature, e.g. [8-9] and references therein. In this sense, the proposed AFC scheme can be applied to quarter-car suspension systems, where its validity and effectiveness can also be demonstrated.

\section{CONCLUSION}

This paper presents a new control design for full-car active suspension systems with unknown nonlinear dynamics. Unlike 
the existing results, the proposed control does not use any function approximation, whilst the unavoidable uncertainties and nonlinearities in the suspension systems can be tackled effectively. This control has a simple proportional-like structure with less requirements on the system and improved computation efficiency. Moreover, both the predefined transient and steady-state suspension motion bounds are strictly guaranteed by incorporating PPFs into the control design. These features make it more suitable for practical application. The stability of the closed-loop control system is rigorously proved by using Extreme Value Theorem and Lyapunov Theorem. A dynamic simulator consisting of commercial software Carsim ${ }^{\circledR}$ and Matlab/Simulink has been built to conduct comparative simulations, where a realistic E-SUV type full-car model embedded in $\mathrm{Carsim}^{\circledR}$ is utilized. Simulation results show the efficacy and superior performance of the proposed control over several other suspension methods. Future work will focus on the validation of the proposed AFC schemes on practical active suspension systems including the actuator dynamics.

\section{APPENDIX}

Proof: The proof follows a similar procedure as [31], which consists of three steps. We first prove the existence of the maximal solution $\zeta(t)$ of (28) over the set $\Omega_{\zeta}$ for a time interval $\left[0, \tau_{\max }\right)$. Then, we prove the boundedness of the closed-loop system signals with the proposed controls (13) and (17) for all $t \in\left[0, \tau_{\max }\right)$. We finally prove that $\tau_{\max }=\infty$ holds for all $\zeta(t) \in \Omega_{\zeta}, \forall t \in\left[0, \tau_{\max }\right)$.

Step 1. It has been stated that the PPF parameters can be set to guarantee the initial conditions $\left|x_{1}(0)\right|<\delta \varphi_{10},\left|e_{1}(0)\right|<\delta \varphi_{20}$. Then the fact $\left|\zeta_{k}(0)\right|<\delta, k=1,2$ is true, i.e. $\zeta(0) \in \Omega_{\zeta}$. Moreover, since the suspension dynamics in (4) and the PPFs $\varphi_{k}(t)$ are continuous with respect to their coordinates, the function $v$ in (28) is piecewise continuous, locally Lipschitz on $\zeta$ over the set $\Omega_{\zeta}$, and piecewise continuous on $t$ for each fixed $\zeta(t) \in \Omega_{\zeta}$. Hence, based on Theorem 1, there exists a maximal solution $\xi(t)$ of (28) on a time interval $t \in\left[0, \tau_{\max }\right)$, such that $\zeta_{i}(t) \in(-\delta, \delta), i=1,2, \forall t \in\left[0, \tau_{\max }\right)$.

Step 2. To prove the boundedness of all closed-loop control system signals for all $t \in\left[0, \tau_{\max }\right)$ with the proposed control, we will calculate the time derivative of $\varepsilon_{k}, k=1,2$ along (14) and (18) with (26) and (27) as:

$$
\begin{aligned}
\dot{\varepsilon}_{1}= & \frac{\partial S^{-1}}{\partial \zeta_{1}} \dot{\zeta}_{1}=r_{1}\left(\dot{x}_{1}-\zeta_{1} \dot{\varphi}_{1}\right)=r_{1}\left(\zeta_{2} \varphi_{2}-k_{1} \varepsilon_{1}-\zeta_{1} \dot{\varphi}_{1}\right) \\
\dot{\varepsilon}_{2}= & \frac{\partial S^{-1}}{\partial \zeta_{2}} \dot{\zeta}_{2}=r_{2}\left[\frac { 1 } { M } \left(-\sum_{i=1}^{4} F_{s i}\left(\zeta_{1} \varphi_{1}, \zeta_{2} \varphi_{2}+u_{1}, \zeta_{3} \varphi_{3}, \zeta_{4} \varphi_{4}+u_{3}, \zeta_{5} \varphi_{5},\right.\right.\right. \\
& \left.\zeta_{6} \varphi_{6}+u_{5}, \bar{x}\right)-\sum_{i=1}^{4} F_{d i}\left(\zeta_{1} \varphi_{1}, \zeta_{2} \varphi_{2}+u_{1}, \zeta_{3} \varphi_{3}, \zeta_{4} \varphi_{4}+u_{3},\right. \\
& \left.\left.\left.\zeta_{5} \varphi_{5}, \zeta_{6} \varphi_{6}+u_{5}, \bar{x}\right)+u_{z}\right)-\dot{u}_{1}-\zeta_{2} \dot{\varphi}_{2}\right]
\end{aligned}
$$

where the variables $r_{1}=1 /\left[\varphi_{1}\left(\delta-\zeta_{1}^{2}\right)\right]$ and $r_{2}=1 /\left[\varphi_{2}\left(\delta-\zeta_{2}^{2}\right)\right]$ are bounded by positive constants $r_{M k}>0, k=1,2$, i.e. $0<r_{k} \leq r_{M k}$ is true [28].
We select a Lyapunov function as $V_{1}=\varepsilon_{1}^{2} / 2$, and calculate its time derivative along (29) as:

$$
\dot{V}_{1}=\dot{\varepsilon}_{1}=\varepsilon_{1} r_{1}\left(\zeta_{2} \varphi_{2}-\zeta_{1} \dot{\varphi}_{1}-k_{1} \varepsilon_{1}\right)
$$

Consider $\left|\zeta_{k}(t)\right|<\delta, \forall t \in\left[0, \tau_{\max }\right)$ and the facts that $\dot{\varphi}_{1}, \varphi_{2}$ are all bounded, then by recalling the Extreme Value Theorem [33], we can verify that

$$
\left|\zeta_{2} \varphi_{2}-\zeta_{1} \dot{\varphi}_{1}\right| \leq F_{1} \text {, for } \forall t \in\left[0, \tau_{\max }\right)
$$

is true for a positive constant $F_{1}>0$. Thus, it follows from (30) $-(31)$ :

$$
\dot{V}_{1} \leq\left|\varepsilon_{1}\right| r_{M 1}\left(F_{1}-k_{1}\left|\varepsilon_{1}\right|\right)
$$

Consequently, one can conclude that $\dot{V}_{1}$ is negative when $\left|\varepsilon_{1}\right|>F_{1} / k_{1}$. Then based on the Lyapunov theorem, the variable $\varepsilon_{1}$ will ultimately converge to a set defined by $\Omega_{1}:=\left\{\varepsilon_{1}|| \varepsilon_{1} \mid \leq \varepsilon_{M 1}=\max \left\{\left|\varepsilon_{1}(0)\right|, F_{1} / k_{1}\right\}\right\} \quad$ for $\quad \forall t \in\left[0, \tau_{\max }\right)$. Moreover, the boundedness of $\dot{\varepsilon}_{1}$ can be verified from (29), and the derivative of the first virtual control $\dot{u}_{1}$ is also bounded. Then it can be claimed that $\varepsilon_{1}, \dot{\varepsilon}_{1}, u_{1}, \dot{u}_{1} \in L_{\infty}$ for $t \in\left[0, \tau_{\max }\right)$.

On the other hand, consider the relationship between $\varepsilon_{1}$ and $\zeta_{1}$ given by (11), we have

$$
e^{2 \varepsilon_{1}}=\frac{\delta+\zeta_{1}}{\delta-\zeta_{1}}
$$

Consequently, it can be derived that

$$
-\delta<\frac{e^{-2 \varepsilon_{M 1}} \delta-\delta}{e^{-2 \varepsilon_{M 1}}+1} \leq \zeta_{1}(t) \leq \frac{e^{2 \varepsilon_{M 1}} \delta-\delta}{e^{2 \varepsilon_{M 1}}+1}<\delta
$$

Therefore, we know $-\delta<x_{1} / \varphi_{1}<\delta$ is true, which indicates $-\delta \varphi_{1}(t)<x_{1}(t)<\delta \varphi_{1}(t)$, i.e. the vertical displacement $x_{1}$ is retained within the bound (8) for $t \in\left[0, \tau_{\max }\right)$.

Following the above analysis, we set a Lyapunov function $V_{2}=\varepsilon_{2}^{2} / 2$, then calculate $\dot{V}_{2}$ from (29) as:

$$
\begin{aligned}
\dot{V}_{2}= & \varepsilon_{2} r_{2}\left[\frac { 1 } { M } \left(-\sum_{i=1}^{4} F_{s i}\left(\zeta_{1} \varphi_{1}, \zeta_{2} \varphi_{2}+u_{1}, \zeta_{3} \varphi_{3}, \zeta_{4} \varphi_{4}+u_{3}, \zeta_{5} \varphi_{5},\right.\right.\right. \\
& \left.\zeta_{6} \varphi_{6}+u_{5}, \bar{x}_{i}\right)-\sum_{i=1}^{4} F_{d i}\left(\zeta_{1} \varphi_{1}, \zeta_{2} \varphi_{2}+u_{1}, \zeta_{3} \varphi_{3}, \zeta_{4} \varphi_{4}+u_{3},\right. \\
& \left.\left.\zeta_{5} \varphi_{5}, \zeta_{6} \varphi_{6}+u_{5}, \bar{x}_{i}\right)-\frac{1}{M} k_{2} \varepsilon_{2}-\dot{u}_{1}-\zeta_{2} \dot{\varphi}_{2}\right] \\
& \leq\left|\varepsilon_{2}\right| r_{M 2}\left[F_{2}-k_{2} \theta_{1 \min }\left|\varepsilon_{2}\right|\right]
\end{aligned}
$$

Similar to the arguments given in (31), we know that $\zeta_{i}, \varphi_{i}, \dot{\varphi}_{i}, u_{3}, \dot{u}_{1}, i=1,2$ are all bounded for $t \in\left[0, \tau_{\max }\right)$. Thus, there exists a positive constant $F_{2}>0$ such that

$$
\begin{aligned}
& \mid \frac{1}{M}\left(-\sum_{i=1}^{4} F_{s i}\left(\zeta_{1} \varphi_{1}, \zeta_{2} \varphi_{2}+u_{1}, \zeta_{3} \varphi_{3}, \zeta_{4} \varphi_{4}+u_{3}, \zeta_{5} \varphi_{5}, \zeta_{6} \varphi_{6}+u_{5}, \bar{x}_{i}\right)-\right. \\
& \sum_{i=1}^{4} F_{d i}\left(\zeta_{1} \varphi_{1}, \zeta_{2} \varphi_{2}+u_{1}, \zeta_{3} \varphi_{3}, \zeta_{4} \varphi_{4}+u_{3}, \zeta_{5} \varphi_{5}, \zeta_{6} \varphi_{6}+u_{5}, \bar{x}_{i}\right)-\dot{u}_{1}-\zeta_{2} \dot{\varphi}_{2} \mid \leq F_{2}
\end{aligned}
$$

For $\forall t \in\left[0, \tau_{\max }\right)$, which can be validated by recalling the Extreme Value Theorem and the fact $\left|\zeta_{2}(t)\right|<\delta$. Therefore, we conclude from (35) and the facts $0<r_{2} \leq r_{M 2}$ and $\theta_{1 \min } \leq 1 / M \leq \theta_{1 \max }$ that $\dot{V}_{2} \leq\left|\varepsilon_{2}\right| r_{M 2}\left[F_{2}-k_{2} \theta_{1 \min }\left|\varepsilon_{2}\right|\right]$, which implies that $\varepsilon_{2}$ ultimately converges to the set defined by $\Omega_{2}=\left\{\varepsilon_{2}|| \varepsilon_{2} \mid \leq \varepsilon_{M_{2}}=\max \left\{\left|\varepsilon_{2}(0)\right|, F_{2} /\left(k_{2} \theta_{1 \min }\right)\right\}\right\} \quad$ for $t \in\left[0, \tau_{\max }\right)$. 
Thus, the control signal $u_{z}$ and the state variable $x_{2}$ are all bounded for $t \in\left[0, \tau_{\max }\right)$. Moreover, the boundedness of $\dot{\varepsilon}_{2}$ and $\dot{u}_{z}$ can be easily verified from (29) and (17). Again, similar to (33), we know $e^{2 \varepsilon_{2}}=\left(1+\zeta_{2}\right) /\left(1-\zeta_{2}\right)$, and thus it can be derived that

$$
-\delta<\frac{e^{-2 \varepsilon_{M 2}} \delta-\delta}{e^{-2 \varepsilon_{M 2}}+1} \leq \zeta_{2}(t) \leq \frac{e^{2 \varepsilon_{M 2}} \delta-\delta}{e^{2 \varepsilon_{M 2}}+1}<\delta
$$

Therefore, the fact $-\delta \varphi_{2}(t)<e_{1}(t)<\delta \varphi_{2}(t)$ is also true for $t \in\left[0, \tau_{\max }\right)$.

Step 3. The last step will validate that $\tau_{\max }=\infty$ is true. Equations. (34) and (36) show that the condition $\zeta(t) \in \bar{\Omega}_{\zeta}, \forall t \in\left[0, \tau_{\max }\right)$ holds for a nonempty open set $\bar{\Omega}_{\zeta}=\prod_{k=1}^{2}\left[\frac{e^{-\varepsilon_{M k}} \delta-\delta}{e^{-\varepsilon_{M k}}+1}, \frac{e^{\varepsilon_{M k}} \delta-\delta}{e^{\varepsilon_{M k}}+1}\right]$. Consequently, we can easily verify that $\bar{\Omega}_{\zeta} \subset \Omega_{\zeta}$ for $\Omega_{\zeta}=(-\delta, \delta) \times(-\delta, \delta)$. If we assume $\tau_{\max }<+\infty$ is true, Proposition 1 implies that there exists a finite time $t_{1} \in\left[0, \tau_{\max }\right)$ such that $\zeta\left(t_{1}\right) \notin \bar{\Omega}_{\zeta}$. This claim leads to a contradiction. Therefore, we can conclude that $\tau_{\max }=\infty$ is true. Thus all the closed-loop signals in control system are bounded for $t \geq 0$. Specifically, the vertical motion $x_{1}$ is retained within the predefined bound (8) for $t \geq 0$. This completes the proof.

\section{REFERENCES}

[1] D. Hrovat, "Optimal active suspension structures for quarter-car vehicle models," Automatica, vol. 26, pp. 845-860, 1990.

[2] T. D. Le, M. T. N. Bui, and K. K. Ahn, "Improvement of Vibration Isolation Performance of Isolation System Using Negative Stiffness Structure," IEEE/ASME Trans. Mechatron, vol. 21, pp. 1561-1571, 2016.

[3] X. Tang, H. Du, S. Sun, D. Ning, Z. Xing, and W. Li, "Takagi-Sugeno fuzzy control for semi-active vehicle suspension with a magnetorheological damper and experimental validation," IEEE/ASME Trans. Mechatron, vol. 22, pp. 291-300, 2017.

[4] H. Zhang, X. Zheng, H. Yan, C. Peng, Z. Wang, and Q. Chen, "Codesign of Event-Triggered and Distributed Hळ Filtering for Active Semi-Vehicle Suspension Systems," IEEE/ASME Trans. Mechatron, vol. 22, pp. 1047-1058, 2017.

[5] H. Li, H. Liu, H. Gao, and P. Shi, "Reliable fuzzy control for active suspension systems with actuator delay and fault," IEEE Trans. Fuzzy Syst, vol. 20, pp. 342-357, 2012.

[6] Y. Huang, J. Na, X. Wu, X. Liu, and Y. Guo, "Adaptive control of nonlinear uncertain active suspension systems with prescribed performance," ISA Trans, vol. 54, pp. 145-155, 2015.

[7] H. Li, X. Jing, and H. R. Karimi, "Output-feedback-based $\mathrm{H} \infty$ control for vehicle suspension systems with control delay," IEEE Trans. Ind. Electron, vol. 61, pp. 436-446, 2014.

[8] W. Sun, H. Gao, and O. Kaynak, "Adaptive Backstepping Control for Active Suspension Systems With Hard Constraints," IEEE/ASME Trans. Mechatron, vol. 18, pp. 1072-1079, 2013.

[9] Y. Huang, J. Na, X. Wu, and G. Gao, "Approximation-free control for vehicle active suspensions with hydraulic actuator," IEEE Trans. Ind. Electron, vol. 65, pp. 7258-7267, 2018.

[10] N. Yagiz and Y. Hacioglu, "Backstepping control of a vehicle with active suspensions," Control Eng. Pract, vol. 16, pp. 1457-1467, 2008.

[11] S.-J. Huang and H.-Y. Chen, "Adaptive sliding controller with self-tuning fuzzy compensation for vehicle suspension control," Mechatronics, vol. 16, pp. 607-622, 2006.

[12] I. Fialho and G. J. Balas, "Road adaptive active suspension design using linear parameter-varying gain-scheduling," IEEE Trans. Control Syst. Technol, vol. 10, pp. 43-54, 2002.

[13] Y. Jia, "Robust control with decoupling performance for steering and traction of $4 \mathrm{WS}$ vehicles under velocity-varying motion," IEEE Trans. Control Syst. Technol, vol. 8, pp. 554-569, 2000.
[14] H. Chen, P.-Y. Sun, and K.-H. Guo, "A multi-objective control design for active suspensions with hard constraints," in Proceedings of the 2003 American Control Conference (ACC), 2003, pp. 4371-4376.

[15] L. Zuo, J.-J. Slotine, and S. A. Nayfeh, "Model reaching adaptive control for vibration isolation," IEEE Trans. Control Syst. Technol, vol. 13, pp. 611-617, 2005.

[16] J.-S. Lin and C.-J. Huang, "Nonlinear backstepping active suspension design applied to a half-car model," Veh. Syst. Dyn, vol. 42, pp. 373-393, 2004.

[17] C. Gohrle, A. Schindler, A. Wagner, and O. Sawodny, "Design and vehicle implementation of preview active suspension controllers," IEEE Trans. Control Syst. Technol, vol. 22, pp. 1135-1142, 2014.

[18] H. Li, J. Yu, C. Hilton, and H. Liu, "Adaptive sliding-mode control for nonlinear active suspension vehicle systems using T-S fuzzy approach," IEEE Trans. Ind. Electron, vol. 60, pp. 3328-3338, 2013.

[19] H. Li, X. Jing, H.-K. Lam, and P. Shi, "Fuzzy sampled-data control for uncertain vehicle suspension systems," IEEE Trans. Cybern, vol. 44, pp. 1111-1126, 2014.

[20] M. Wang, B. Chen, and P. Shi, "Adaptive neural control for a class of perturbed strict-feedback nonlinear time-delay systems," IEEE Trans. Syst., Man, Cybern. B, Cybern, vol. 38, pp. 721-730, 2008.

[21] Y.-J. Liu and S. Tong, "Adaptive fuzzy control for a class of unknown nonlinear dynamical systems," Fuzzy Sets and Systems, vol. 263, pp. 49-70, 2015.

[22] J. Na, X. Ren, and D. Zheng, "Adaptive control for nonlinear pure-feedback systems with high-order sliding mode observer," IEEE Trans. Neural. Netw, Learn. Syst, vol. 24, pp. 370-382, 2013.

[23] R. Cui, C. Yang, Y. Li, and S. Sharma, "Adaptive neural network control of AUVs with control input nonlinearities using reinforcement learning," IEEE Trans. Syst., Man, Cybern., Syst., vol. 47, pp. 1019-1029, 2017.

[24] B. Xu, C. Yang, and Z. Shi, "Reinforcement learning output feedback NN control using deterministic learning technique," IEEE Trans. Neural. Netw, Learn. Syst, vol. 25, pp. 635-641, 2014.

[25] J. Na, M. N. Mahyuddin, G. Herrmann, X. Ren, and P. Barber, "Robust adaptive finite - time parameter estimation and control for robotic systems," Int. J. Robust Nonlin. Control, vol. 25, pp. 3045-3071, 2015.

[26] C. P. Bechlioulis and G. A. Rovithakis, "Robust adaptive control of feedback linearizable MIMO nonlinear systems with prescribed performance," IEEE Trans. Autom. Control, vol. 53, pp. 2090-2099, 2008.

[27] C. P. Bechlioulis and G. A. Rovithakis, "Adaptive control with guaranteed transient and steady state tracking error bounds for strict feedback systems," Automatica, vol. 45, pp. 532-538, 2009.

[28] J. Na, Q. Chen, X. Ren, and Y. Guo, "Adaptive prescribed performance motion control of servo mechanisms with friction compensation," IEEE Trans. Ind. Electron, vol. 61, pp. 486-494, 2014.

[29] S. Wang, J. Na, and X. Ren, "RISE-based asymptotic prescribed performance tracking control of nonlinear servo mechanisms," IEEE Trans. Syst., Man, Cybern., Syst., vol. 48, pp. 2359 - 2370, 2018.

[30] J. Na, Y. Huang, X. Wu, G. Gao, G. Herrmann, and J. Z. Jiang, "Active adaptive estimation and control for vehicle suspensions with prescribed performance," IEEE Trans. Control Syst. Technol, pp. 1-15, 2017.

[31] C. P. Bechlioulis and G. A. Rovithakis, "A low-complexity global approximation-free control scheme with prescribed performance for unknown pure feedback systems," Automatica, vol. 50, pp. 1217-1226, 2014.

[32] W. Sun, H. Gao, and B. Yao, "Adaptive Robust Vibration Control of Full-Car Active Suspensions With Electrohydraulic Actuators," IEEE Trans. Control Syst. Technol, vol. 21, pp. 2417-2422, 2013.

[33] E. D. Sontag, Mathematical control theory. London, U.K.: Springer, 1998.

[34] M. Moradi and A. Fekih, "Adaptive PID-sliding-mode fault-tolerant control approach for vehicle suspension systems subject to actuator faults," IEEE Trans. Veh. Technol, vol. 63, pp. 1041-1054, 2014.

[35] S. Ikenaga, F. L. Lewis, J. Campos, and L. Davis, "Active suspension control of ground vehicle based on a full-vehicle model," in Proceedings of the 2000 American Control Conference (ACC), 2000, pp. 4019-4024.

[36] F. Zhao, S. S. Ge, F. Tu, Y. Qin, and M. Dong, "Adaptive neural network control for active suspension system with actuator saturation," IET Control Theory Appl, vol. 10, pp. 1696-1705, 2016. 


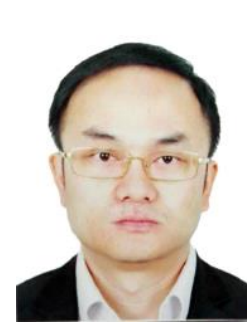

Jing Na (M'15) received the B.Eng. and Ph.D. degrees from the School of Automation, Beijing Institute of Technology, Beijing, China, in 2004 and 2010, respectively.

From 2011 to 2013, he was a Monaco/ITER Postdoctoral Fellow at the ITER Organization,

Saint-Paul-lès-Durance, France. From 2015 to 2017, he was a Marie Curie Intra-European Fellow with the Department of Mechanical Engineering, University of Bristol, U.K. Since 2010, he has been with the Faculty of Mechanical and Electrical Engineering, Kunming University of Science and Technology, Kunming, China, where he became a Professor in 2013. He has coauthored one monograph and more than 100 international journal and conference papers. His current research interests include intelligent control, adaptive parameter estimation, nonlinear control and applications for robotics, vehicle systems and wave energy convertor, etc.

$\mathrm{He}$ is currently an Associate Editor of the IEEE Transactions on Industrial Electronics, the Neurocomputing, and has served as the Organization Committee Chair of DDCLS 2019, international program committee Chair of ICMIC 2017. Dr Na has been awarded the Best Application Paper Award of the 3rd IFAC International Conference on Intelligent Control and Automation Science (IFAC ICONS 2013), and the 2017 Hsue-shen Tsien Paper Award.

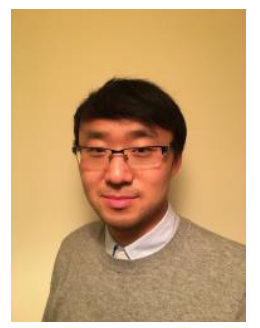

Yingbo Huang (M'19) received the B.Sc. degree from Lanzhou City University, Lanzhou, China, and the Ph.D degree from the Faculty of Mechanical and Electrical Engineering, Kunming University of Science and Technology, Kunming, China, in 2013 and 2019, respextively.

$\mathrm{He}$ is currently a lecture with Faculty of Mechanical and Electrical Engineering, Kunming University of Science and Technology, Kunming, China. His current research interests include adaptive control and transient performance improvement of nonlinear systesms with application to vehicle suspension systems.

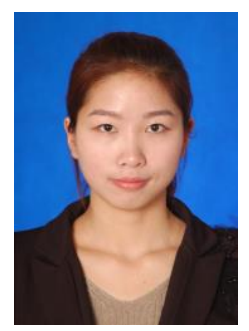

Qianqian Pei received the M.Sc. degree from the Faculty of Mechanical and Electrical Engineering, Kunming University of Science and Technology, Kunming, China in 2017.

She is currently a lecture with Faculty of Mechanical and Electrical Engineering, Yunnan Land And Resources Vocational College, Kunming, China. Her current research interests include adaptive control, parameter estimation and application.

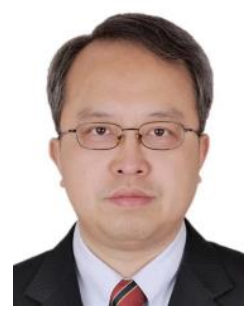

Xing Wu received the B.Sc. and M.Sc. degrees from Kunming University of Science and Technology, China, in 1994 and 1997, respectively, and the Ph.D. degree in mechanical engineering from Shanghai Jiao Tong University, China, in 2005.

$\mathrm{He}$ is currently a Professor with the
Faculty of Mechanical and Electrical Engineering, Kunming University of Science and Technology. His current research interests include modern signal processing theory and their applications on fault feature extracting, and internet-based mechanical fault diagnosis technology and expert systems.

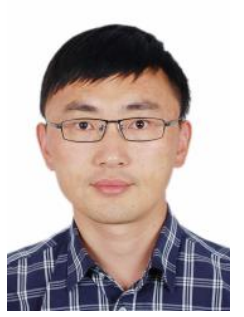

Guanbin Gao received the B.Sc. and M.Sc. degrees in mechanical engineering and automation from Northeastern University, Shenyang, China, in 2001 and 2004, respectively, and the Ph.D. degree in mechanical manufacturing and automation from Zhejiang University, Hangzhou, China, in 2010.

His current research interests include precision measuring \& control, kinematics of industrial robots, and NNs.

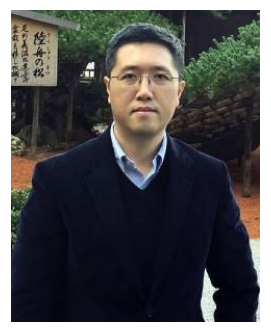

Guang Li (M'09) received the Ph.D. degree in Electrical and Electronics Engineering, specialized in control systems, from the University of Manchester, in 2007.

$\mathrm{He}$ is currently a senior lecturer of dynamics modelling and control with Queen Mary University of London, UK. His current research interests include constrained optimal control, model predictive control, adaptive robust control and control applications including renewable energies and energy storage, etc. 